\title{
Circular Polarized Antenna Design for RFID Logistics Label based on Ultra High Frequency
}

\author{
Jing Wang \\ China Construction Eighth Engineering Bureau workstation \\ of postdoctoral scientific research \\ Shanghai 200135; \\ Management science and Engineering in Shanghai Jiao \\ Tong University post-doctoral research station \\ Shanghai 200240; \\ Northwest University of Politics and School of \\ Economics and Management \\ Xi'an 710063 \\ wjing818@126.com,719428937@qq.com
}

\begin{abstract}
This paper discloses a circularly polarized antenna, ultra high frequency radio frequency identification tag includes: Based on a cylindrical shell, the shell is composed of a casing and a casing, wherein the upper shell is arranged on the inner side of a clamping groove; an antenna control module, are fixedly arranged in the shell; a radio frequency module, arranged in the slot, and the antenna control module is electrically connected; a radio frequency cable, and the RF module is electrically connected, and extends out of the upper shell. Originally in the outside of the shell is welded by means of sealing connection RF cable provided by the utility model, encapsulated into the shell, the shell of the whole sealing, waterproof performance is very excellent at. This product volume is much smaller than other products, can damage to the ground or floor structure decreases when, at the same time ABS shell material for shell of the product, the grinding pressure performance, make the product has better convenient construction, but also improves the signal sensitivity.
\end{abstract}

Keywords-Ultra high frequency radio frequency identification label; logistics; circular polarization antenna

\section{INTRODUCTION}

This paper relates to the technical field is about a practical antenna model, especially a circularly polarized antenna based on ultra high frequency radio frequency identification tag.

\section{THE TECHNICAL BACKGROUND}

There are a lot of wireless communications equipment was used in logistics, warehousing, cargo storage and transfer for monitoring, using the signal transmit antenna excitation and read electronic tag information, to achieve the purpose of monitoring the transfer of goods [1]. But the antenna signal is the range and angle, based on the same power, in order to achieve the best effect of monitoring range and the biggest, people often will antenna buried under the ground, so as to make full use of the signal angle

\author{
Rongquan Ma \\ China Construction Eighth Engineering Bureau workstation \\ of postdoctoral scientific research, Shanghai 200135
}

of antenna, monitoring on the ground can reach 360 degrees, but this kind of buried antenna cannot solve the waterproof the problem of pressure. The application of radio frequency identification (RFID) antenna volume is relatively large, and is square, which will cause the shielding and wireless interference. In addition, the exposed joints waterproof also difficult to deal with, which will affect the stability of RFID system [2].

\section{The UTILITY MODEL}

The purpose of the utility model is to solve the defects existing in the prior art, and provides a small volume, strong compression resistance, good waterproof performance, circular polarization antenna stable work for RFID.

To achieve the above purpose, the utility model provides a circularly polarized antenna for ultra high frequency radio frequency identification tag based, including:

A cylindrical shell, the shell is composed of a shell and a shell, wherein the upper shell is arranged on the inner side of a clamping groove;

An antenna control module, is fixedly arranged on the shell;

A radio frequency module, is arranged in the slot, and the control module is electrically connected to the antenna;

$A$ radio frequency cable, and the RF module is electrically connected, and extends out of the upper shell.

Further, the upper shell and the lower shell are fixedly connected by a screw.

Further, the joint part and screw the upper shell and the lower shell sealing connection through the wax immersion method.

Further, the RF module and the upper shell by wax immersion way of sealing connection.

Further, the upper shell and the lower shell with ABS shell material. 
The utility model based on circular polarization antenna ultra high frequency radio frequency identification tag, ultra high frequency in particular to work in the $900 \mathrm{MHz}$ $-930 \mathrm{MHz}$ range (UHF) radio frequency identification (RFID) system[3]. The cross section of the circular polarization antenna is a circular, the whole cylindrical shell material, including by making ABS a shell and a shell with tuning and a supporting role, and the antenna casing arranged on the lower shell, control module, RF module etc.

Originally in the outside of the shell is welded by means of sealing RF cable connected by the utility model, encapsulated into the shell, the shell of the whole sealing, waterproof performance is very excellent at[4]. This product volume is much smaller than other products, has the advantages of small volume, strong compression resistance, good waterproof performance, stable work, can damage to the ground or floor structure decreases when, at the same time ABS shell material for shell of the product, the product has better wear resistance to pressure performance, convenient construction, but also improve the the signal sensitivity [5].

The utility model has the following advantages: 1 through a coaxial cable is connected directly with the reader, small volume, high integration degree. 2 no wiring, easy installation, encoding settings and inspection or are simple and convenient. 3 information management, transparent, timely, managers at all levels can be through simple network management, inquire into all kinds of production information. The invention is novel in design, easy to use, the market prospect is broad [6]

\section{DESCRIPTION OF FIgURES}

Figure 1 for the utility model circularly polarized antenna for ultra high frequency radio frequency identification tag on the basis of structure sketch map.

Figure 2 for the utility model circularly polarized antenna for ultra high frequency radio frequency identification tag the bend section based on optic structure schematic diagram.

Figure 3 for the utility model circularly polarized antenna for ultra high frequency radio frequency identification tag based on Application in general chip schematic diagram.

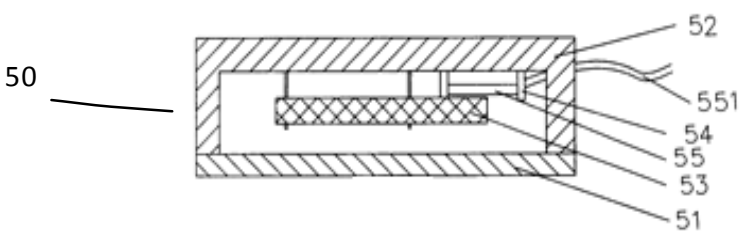

Figure 1.

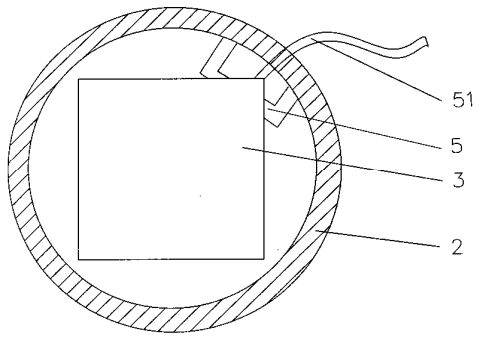

Figure 2.

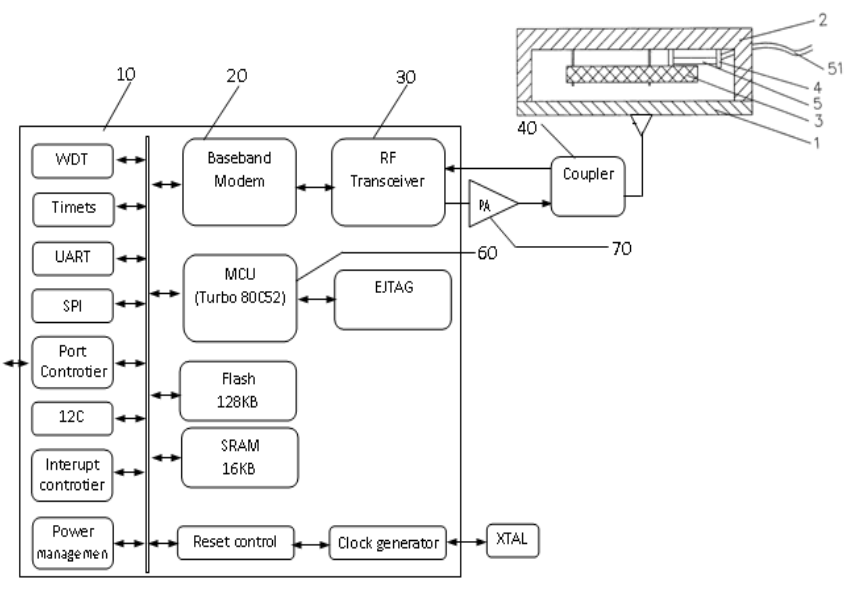

Figure 3.

\section{THE SPECIFIC IMPLEMENTATION WAY}

In order to make the purpose of the utility model, technical solutions and advantages of clear, the following combination of drawings and the embodiment of the utility model, for further details. It should be understood, in particular embodiments described herein is explained by the utility model, is not used to limit the utility model.

See Figure 1 and Figure 2, the utility model based on circular polarization antenna ultra high frequency radio frequency identification tag, including:

A cylindrical shell 50 , the housing 50 by using the ABS shell material of a case body 52 and a housing 51, the upper shell and the lower shell body 51 and 52 are connected by screws, in order to be part of signal transmission device package into the shell 50, the upper shell 52 provided inside with a card slot 54 ;

An antenna control module 53, fixed in the upper shell body 52 ;

A radio frequency module 55, arranged in the card slot 54 , and the 53 antenna control module is electrically connected;

A radio frequency cable 551 , and the RF module 55 electrical connection, and extends out of the upper shell 52 , 
used for the RF module 55 is connected with the external power supply.

Further, the joint part and screw shell 52 and the lower case 51 sealing connection through the wax immersion method. The RF module 55 and the upper shell body 52 through the wax immersion way of sealing connection.

As shown in Figure 3, the utility model is circularly polarized antenna for ultra high frequency radio frequency identification tag based on Application in general chip schematic diagram, in which, the general chip 10 is mainly provided with a micro processing unit (MCU) 60, RF transceiver module 30, modulation and demodulation module 20, the radio frequency transceiver module 30 through the modulation and demodulation module 20 and a micro processing unit (MCU) 60 is connected, the micro processing unit (MCU) 60 through the data bus and memory (fash memory), cache (SRAM), reset control circuit (Reset control), a watchdog timer circuit (WDT), a timer (Timers), universal asynchronous receiver / transmitter (UART), serial peripheral interface (SPI), port controller (Port controller), serial bus (12C), interrupt controller (Interupt controller) and power management (power management) connection. RF transceiver module 30 are respectively provided with a transmitting port and receive port, the port and the receiving port by the power amplifier 70 and 40 connected coupler, coupler 40 then the communication signal is transmitted to the utility model of the antenna control module 53 [7].

The combination of the above the embodiment of the utility model is described in detail, the general technical staff in this field according to the instructions of the utility model, make all sorts of changes[8]. Therefore, should not constitute a restriction on the utility model implementation details in some cases, the utility model in the appended claims to define the scope of protection range of the utility model as.

\section{CONCLUSION}

The utility model relates to a super high frequency (UHF) radio frequency identification (RFID) tag of the circularly polarized antenna, ultra high frequency and particularly relates to a work in the $900 \mathrm{MHz}-930 \mathrm{MHz}$ range (UHF) radio frequency identification (RFID) system[9]. Circularly polarized antenna for the UHF RFID tag, the overall shape of a pie, including ABS shell material has tuning and a supporting role, and arranged on the lower shell, ABS shell material on the shell, the antenna module etc.. Circular polarization antenna UHF RFID tag at least one end and general chip external circuit welding block connection and circularly polarized antenna part is fixed to the RFID tag based on UHF substrate. Because the UHF RFID tag using multiple short UHF RFID circularly polarized antenna instead of linear polarization antenna, for linear polarization label, in the scope of radiation circularly polarized antenna, planar antenna is on the label can be arbitrarily rotated it will not affect the reading. The circularly polarized antenna, linear polarization label due to polarization match will lose the general received power, which makes the reading distance decreased[10]. So because of the small volume can be as much as possible the integrated tag, and can quickly and easily implement the antenna coupled to the process on a chip. Circular polarization antenna based on UHF RFID tag is made by ABS material of shell, the pattern of the antenna is determined by the antenna structure, once the antenna structure determination, will determine the direction map. The gain of the antenna with half wave power beam width is restrict each other, gain is big, the beam width is smaller, and vice versa. Circular polarization antenna UHF RFID tag based on good in Bobbi in the frequency range, show that the antenna has good working performance [11].

The utility model has the advantages of small volume, strong compression resistance, good waterproof performance, stable work. The invention has the advantages as follows: 1 the innovation through a coaxial cable is connected directly with the reader, small volume, high integration degree. 2 no wiring, easy installation, encoding settings and inspection or are simple and convenient. 3 information management, transparent, timely, managers at all levels can be through simple network management, inquire into all kinds of production information. The invention is novel in design, easy to use, the market prospect is broad[12].

\section{ACKNOWLEDGEMENTS}

In 2010 the National Social Science Fund Project, number10BJY075. 2, China Postdoctoral Science Foundation funded project, number2012T50796. 3, The 2012 annual Xi'an Municipal Science and Technology Bureau of industrial technology innovation project, numberCX1255.

\section{REFERENCES}

[1] Wang L C,Lin Y C,Lin P H.Dynamic mobile RFID-based supplychain control and management system in construction $[\mathrm{J}]$.AdvancedEngineering Informatics, 2007,21(4): 377-390

[2] Patrick J,Sweeney II.RFID For Dummies[M].Wiley Publishing,Ine.2005: 205-213.

[3] Fifteen ministry of the People's Republic of China ministry of science and technology, etc. China radio frequency identification (RFID) technology policy white paper [R]. National ministry of science and technology, national development and reform commission, ministry of commerce, ministry of information industry and other 15 ministries and other ministries, 15, 2006-06-09

[4] zhang bin, Yao Dagong. RFID technology in the application of real-time logistics [J]. Value engineering, 2004:113-116

[5] Ceng Jiang OuYangYu. Radio frequency identification (rfid) and electronic tag $[\mathrm{M}]$. Beijing: China economic publishing house, 2005:165

[6] Nakib F.Technological adaptability,an approach toward aflexibleand sustainable architecture[C]/ /Conference on Technology \& Sustainability in the Built Environment,2010: 479-494

[7] A Verroust,F Schonek,D Roller.Rule-oriented method for parameterized computer-aided design [J].Computer- Aided Design, 1992, 24(10): 531-540.

[8] LanLiHui. Discuss about parametric design method of CAD products $[\mathrm{J}]$. Journal of changchun college of engineering, 2003, 23 (3) : 70-72. 
[9] Wang Junze QuChang. Based on the network standard three-dimensional parameterized simulation $[\mathrm{J}]$. Journal of machine tools and hydraulic, 2010, 26 (5) : 35-39.

[10] okamoto, jin, ikeda is tree. An automated build system for $3 \mathrm{~d}$ model: Japan, CN200580007187.7 [P]. 2007-03-14.

[11] Wu Xinyuan ZhangXiangFeng, Guo Lianshui. 3 d parametric CAD system research and implementation of product platform for building a general $[\mathrm{J}]$. Journal of mechanical manufacturing, 2001, 33 (2) 6:7-9.

[12] $\mathrm{Wu}$ Tao, double clear soup. The top-down design oriented $3 \mathrm{~d}$ feature modeling technology research $[\mathrm{J}]$. Journal of huazhong university of science and technology (natural science edition), 2003, 31 (8): 1-3. 